\title{
Wertschöpfungspartnerschaften in der Immobilienprojektentwicklung
}

Empirische Analyse der Erfolgswirkungen

\author{
Kevin Meyer $\cdot$ Andreas Pfnür
}

Online publiziert: 24. Juni 2015

(C) Die Autor(en) 2015. Dieser Artikel ist auf Springerlink.com mit Open Access verfügbar.

Zusammenfassung Dieser Beitrag untersucht, inwieweit partnerschaftliche Organisationsmodelle bei der Projektentwickung, wie sie in grundsätzlich ähnlicher Form bei ÖPPs allerdings in einem anderen Handlungsrahmen schon seit über einem Jahrzehnt zur Anwendung kommen, zur Reduzierung von Ineffizienzen bei der Immobilienbeschaffung privatwirtschaftlicher Unternehmen beitragen kann und was die Erfolgsdeterminanten dieser alternativen Beschaffungsvariante sind. Hierzu wurden zwei Pilotprojekte, wissenschaftlich begleitet und mit einem Mix aus qualitativen und quantitativen Methoden wirkungsanalytisch untersucht. Aus der empirischen Analyse geht hervor, dass in beiden Pilotprojekten durch die Wertschöpfungspartnerschaften die Effizienz der Projektentwicklung erheblich erhöht werden konnte. Höheres Vertrauen und größere Motivation der Projektbeteiligten haben positive Einflüsse auf die Bereitschaft, Informationen und Wissen zur Optimierung des Projekterfolges auszutauschen. Auffallend sind allerdings die unterschiedlichen Ausprägungen dieser Einflussfaktoren in beiden Pilotprojekten. Die Ursache für die unterschiedlichen Vertrauensverhältnisse und den entsprechend ungleichverteilten Effekte liegt vor allem in den abweichenden vertraglichen Strukturen innerhalb der Projekte. Der Beitrag zeigt theoretisch konzeptionelle sowie anhand der Fallstudien, wie sich unterschiedliche Projektstrukturen auf die Zusammenarbeit auswirken. Da es sich im empirischen Teil vom Forschungsansatz her um Fallstudien handelt, ist die Allgemeingültigkeit der Ergebnisse naturgemäß limitiert.

\footnotetext{
A. Pfnür $(\bowtie) \cdot K$. Meyer

Fachgebiet Immobilienwirtschaft und Baubetriebswirtschaftslehre der Technischen Universität

Darmstadt,

Darmstadt, Deutschland

E-Mail: pfnuer@bwl.tu-darmstadt.de

K. Meyer

E-Mail: meyer@bwl.tu-darmstadt.de
} 
Schlüsselwörter Immobilienprojektentwicklung · Projektmanagement ·

Wertschöpfungspartnerschaften · Immobilienbereitstellung · Betriebliche immobilienwirtschaft

\section{Value added partnerships in real estate development}

Empirical analysis of the performance effects

Abstract For non-property companies and their core business transformation process, an important input-resource is space, respectively real estate. Due to huge capital and operating expense requirements of real estate as well as its influence on corporate productivity, procuring real estate and real estate services impacts company performance significantly. As real estate developments and acquisitions very often show massive overruns in time and budget as well as quality deficiencies, real estate procurement needs innovation. Therefore, the public sector has developed and, in more and more instances, used the concept of PPP (Public Private Partnerships), thus increasing efficiency of their real estate projects.

In scientific investigations, a lack of cooperation and poor communication between the project stakeholders were repeatedly identified as potential causes of these inefficiencies. Therefore deals this paper with the question, which success factors contribute to a functioning and active cooperation- and communication behaviour within real estate development projects and how these also affect the quality and efficiency of projects. For this purpose, two projects of value-added partnerships in the real estate industry were scientifically examined during the planning- and realisation phase, by using a mixture of qualitative- and quantitative methods. The projects concerned the newly built head offices of medium-sized corporates, which have both been realised as value-added partnerships. Already completed examinations however, show clear differences in the achievement of their targets: Project A was fulfilled within the budgeted costs and the scheduled time frame; Project B, on the other hand, required a significant budget- and schedule amendment already during preliminary design. Moreover, a massive dissatisfaction with the functional quality of the office space was found by a pre- and post survey amongst the employees in project A. These different efficiency enhancement effects of value-added partnerships appear in particular in public-private partnerships (PPPs) in public building. This procurement method is in the public sector, therefore, controversial. Empiric analyses with regard to their favourableness are evaluated very critically. To date, there are no analytic investigations as to the effects of value-added partnerships in the real-estate industry, that do not only show the monetary effects, but do also relate these to their causes. The subsequent analysis is supposed to close this gap. Due to the fact that the public sector was very hesitant in providing empirical data, this paper analyses the basic mechanisms exclusively on the base of private valueadded partnerships. The purposes of this analysis are the identification of causal effects on success of the innovative procurement option and the identification of the determinants of success. The findings gained allow for a responsible real estate management to conclude that besides the life-cycle-oriented organisational form, functioning social relationships among the project's network are crucial. Further- 
more, it should be considered that the requirements may vary with different user types. Therefore, it is wise to enhance the relationships between the specific project actors according to the user's requirements, in order to stimulate the self-determination and the intensity of cooperation of the organisation respectively, to ultimately favour an ideal project result. Thus, users aiming to optimise efficiency for instance should pay special attention to strong relationships between the value-added steps of planning, building and operation. Users focusing on ideal working conditions should, in addition to professionally integrating the user's interests, take care of a close relationship of planning and users.

Keywords Real estate development · Project management · Value-addedpartnerships $\cdot$ Property supply $\cdot$ Corporate real estate management

\section{Einleitung}

Die Beschaffung von Immobilien als Betriebsmittel von Unternehmen hat aufgrund der hiermit verbundenen Kapital- und Betriebskosten dieser Ressourcenart eine besondere Relevanz für den Unternehmenserfolg (Pfnür 2002, S. 29 f.). Allerdings sind bei Immobilienprojekten immer wieder massive Zeit- und Kostenüberschreitungen sowie erhebliche Qualitätsmängel zu beobachten, woraus sich ein erhebliches Risikopotential für die Unternehmen ergibt. Als potentielle Ursachen dieser Ineffizienzen wurden in wissenschaftlichen Untersuchungen immer wieder eine fehlende Kooperationsbereitschaft und eine mangelhafte Kommunikation der beteiligten Projektakteure identifiziert (Pinto und Mantel 1990, S. 274). Dieser Beitrag geht der Frage nach, was die Erfolgsfaktoren für ein funktionierendes und aktives Kooperations- und Kommunikationsverhalten innerhalb von Immobilienprojektentwicklungen sind und wie dadurch die Qualität und die Effizienz von Projekten beeinflusst werden kann. Hierfür wurden zwei Projekte immobilienwirtschaftlicher Wertschöpfungspartnerschaften über die Planungs- und Realisierungsphase hinweg wissenschaftlich begleitet und mit einem Mix aus qualitativen und quantitativen Methoden untersucht.

Bei den Projekten handelt es sich um die Neubauten der Unternehmenszentralen zweier mittelständischer Unternehmen, die beide als Wertschöpfungspartnerschaften realisiert wurden. Allerdings zeigen bereits abgeschlossene Evaluationen wirtschaftlicher und qualitativer Zielvorgaben deutliche Unterschiede zwischen beiden Projekten. Variierende Effizienzsteigerungswirkungen von Wertschöpfungspartnerschaften zeigen sich insbesondere auch bei Öffentlich Privaten Partnerschaften (ÖPPs) im öffentlichen Hochbau. Infolge dessen ist diese Beschaffungsvariante für die öffentliche Hand bislang auch umstritten und empirische Analysen zu deren Vorteilhaftigkeit werden sehr kritisch beurteilt (Bundesrechnungshof 2011). Es existieren bis zum jetzigen Zeitpunkt keine wirkungsanalytischen Untersuchungen von immobilienwirtschaftlichen Wertschöpfungspartnerschaften, die nicht nur die monetären Effekte zeigen, sondern diese auch kausal auf ihre Ursachen zurückführen. Diese Lücke soll mit der nachfolgenden Analyse geschlossen werden. Aufgrund der Tatsache, dass die öffentliche Hand mit der Bereitstellung empirischer Daten sehr zurückhaltend war, 


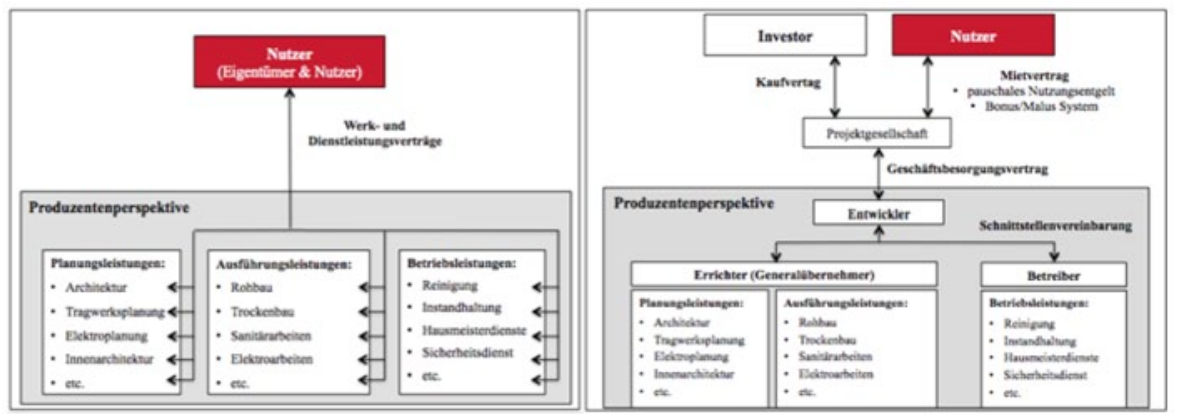

Abb. 1 Organisationsschemata einer konventionellen Beschaffungsvariante und einer Wertschöpfungspartnerschaft. (Eigene Darstellung)

werden die grundlegenden Mechanismen in diesem Beitrag anhand von rein privatwirtschaftlichen Wertschöpfungspartnerschaften analysiert. Die Zwecke der Analyse sind die Identifikation von kausalen Erfolgswirkungen dieser innovativen Beschaffungsvariante und die Identifikation der Erfolgsdeterminanten.

\section{Theoretischer Bezugsrahmen und Hypothesenbildung}

\subsection{Grundzüge immobilienwirtschaftlicher Wertschöpfungspartnerschaften}

Immobilienwirtschaftliche Wertschöpfungspartnerschaften, wie alle beschaffungsorientierten Netzwerke per se, sind eine spezifische Art von „Wertschöpfungssystemen“. Unter dem Begriff „Wertschöpfungssystem“ wird im Allgemeinen ein abgestimmtes Handeln zur Erzeugung von Nutzen verstanden (Heuskel 1999, S. 36).

Immobilienwirtschaftliche Wertschöpfungspartnerschaften fallen in der Managementlehre in die Gruppe der strategischen Netzwerke und zeichnen sich durch komplexe, netzwerkartige, vertrauensbasierte Strukturen zur gemeinschaftlichen Aufgabenerledigung zwischen Bedarfsträgern und Lieferanten entlang der gesamten Wertschöpfungskette aus (Johnston und Lawrence 1998, S. 195).

Die Abb. 1 zeigt anhand der vereinfachten Organisationsschemata die Unterschiede einer konventionellen Variante der Beschaffung immobiliarer Betriebsmittel und einer Wertschöpfungspartnerschaft.

Ein wesentlicher Unterschied liegt darin, dass bei einer Wertschöpfungspartnerschaft die Leistungserstellung von einer zentralen Organisation ganzheitlich koordiniert und verantwortet wird. Ein Entwickler schließt sich dabei in der Regel mit einem Generalübernehmer, welcher die Planung und den Bau verantwortet und einem Betreiber, der alle betriebsrelevanten Leistungen abdeckt, zusammen. So erfolgt eine integrale Planung, bei der bereits während der Planungsphase die Anforderungen des Betriebes und der Nutzung Berücksichtigung finden. Das beauftragende Unternehmen erhält somit alle Planungs-, Ausführungs- und Betreiberleistungen aus einer Hand. In der konventionellen Beschaffungsvariante hingegen, wird die Leistung durch Einzelvergabe meist von einzelnen unabhängigen Organisationen erbracht, ohne zentrale Verantwortung und ohne frühzeitige Einbeziehung von Betriebskom- 
petenzen. Alternativ wird häufig von der Vergabe an einen Generalunternehmer gebrauch gemacht, der die Immobilie schlüsselfertig erstellt (Girmscheid 2007). Weil die Unterschiede in den Anreiz- und Kommunikationsstrukturen in diesen beiden Fällen am größten sind, soll zu didaktischen Zwecken nachfolgend die Einzelvergabe als die konventionelle Beschaffungsvariante der Wertschöpfungspartnerschaft gegenübergestellt werden.

Im Rahmen einer Wertschöpfungspartnerschaft wird zu Beginn des Projektes die vom Nutzer nachgefragte Leistung mit funktional ergebnisorientierten Leistungsbeschreibungen definiert. So wird gewährleistet, dass die verschiedenen Akteure der Produzentenperspektive ihr Know-how zur Entwicklung effizienter Lösungsansätze bestmöglich einbringen können. Sofern die langfristige Finanzierung durch einen externen Investor erfolgt, schließt das nutzende Unternehmen lediglich einen Mietvertrag ab, der neben der Miete auch alle betriebsbedingten Kosten in einem pauschalen Nutzungsentgelt inkludiert. Mögliche Kostensteigerungsrisiken im Betrieb werden dadurch auf den Betreiber übertragen, der die im Nutzungsentgelt vereinbarte Obergrenze der Betriebskosten garantiert. Dieser Risikoübertrag stellt einen wesentlichen Unterschied gegenüber einer Vergabe an einen Generalunternehmer dar. In der Regel werden die Betriebskosten für den Immobiliennutzer in einem Generalunternehmermodell nicht pauschaliert.

Die Projektrisiken liegen bei einer immobilienwirtschaftlichen Wertschöpfungspartnerschaft somit bei den Akteuren der Produzentenperspektive und werden demjenigen Partner übertragen, der das Risiko am besten beherrscht. Die Vergütung der Partner erfolgt grundsätzlich leistungsorientiert durch Bonus und Malus Regelungen. Wirtschaftliche Abhängigkeiten, wie beispielsweise die Entscheidung für höherwertige Materialien oder Systeme zur Reduzierung von Betriebskosten, werden zwischen den Wertschöpfungsstufen durch entsprechende Schnittstellenvereinbarungen geregelt. Es resultiert ein integriertes Planungs- und Steuerungskonzept von Planung, Bau und Betrieb sowie Finanzierung, durch das alle Projektbeteiligte vom ersten Tag an gemeinsame Optimierungspotenziale erarbeiten. Im Unterschied zu reinen lebenszyklusorientierten Planungskonzepten, wird hier durch entsprechende Anreizund Vergütungssysteme auch für die Durchsetzung der Lebenszyklusoptimierung gesorgt (Pfnür 2011, S. 436 ff). Aus diesen wirtschaftlichen Abhängigkeiten ergeben sich Zielkongruenzen, die das Vertrauen und damit das soziale Kapital innerhalb des Netzwerkes stärken und damit die Basis für ein aktives Kommunikations- und Kooperationsverhalten zwischen den Beteiligten bilden (Budäus und Grüb 2007, S. 258).

Nachfolgend ist nun zu untersuchen, wie sich die auf Vertrauen basierende Koordination einer Wertschöpfungspartnerschaft auf das Kommunikations- und Kooperationsverhalten der Beteiligten auswirkt und welche Konsequenzen sich für den jeweiligen Projekterfolg ergeben.

\subsection{Modellbildung}

Die zentrale Voraussetzung der Identifikation und Bewertung kausaler Zusammenhänge zwischen der Organisation von Immobilienprojektentwicklungen und dem Erfolg, ist zunächst die modellhaft vereinfachte Abbildung der Realität. Das hier ver- 


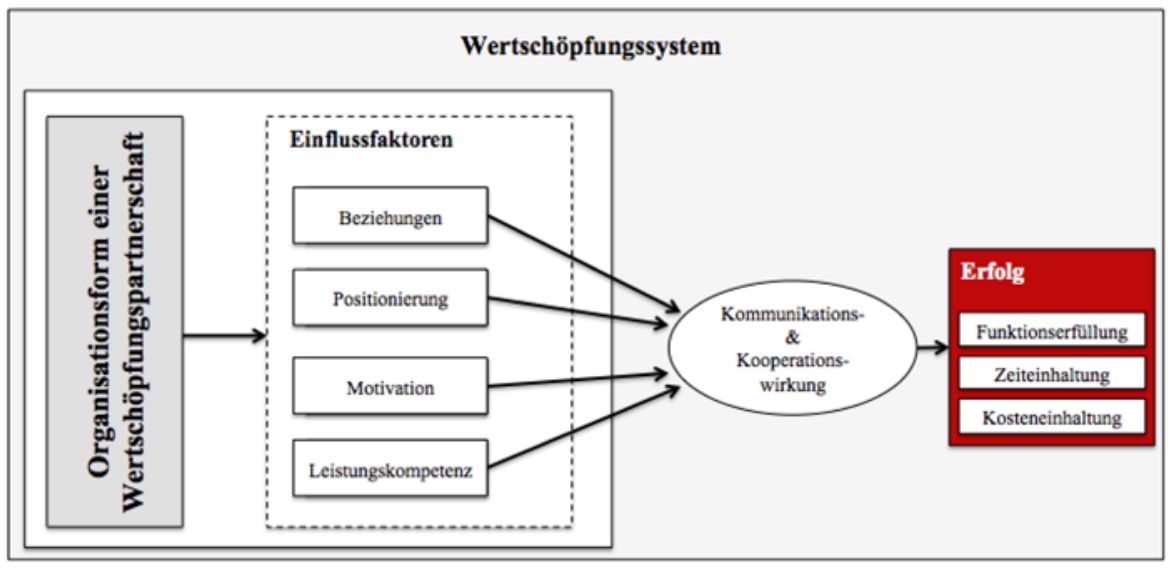

Abb. 2 Theoretischer Bezugsrahmen. (Eigene Darstellung)

wendete Modell besteht aus drei zentralen Konstrukten: (Möller 2006, S. 185). Die Organisationsform bildet quasi die Hülle zur Ausgestaltung der Wertschöpfungspartnerschaft, in der Aufgaben und Verantwortungen zugeordnet und Steuerungssysteme festgelegt werden sowie vertragliche Strukturen geregelt sind. Unter dem zweiten Konstrukt, den Einflussfaktoren, werden Faktoren verstanden, die das Agieren der Mitglieder der Wertschöpfungspartnerschaft betreffen und direkten Einfluss auf die Kommunikations- und Kooperationsbereitschaft der beteiligten Organisationen haben können und regelmäßig als zentrale Faktoren für eine erfolgreiche Kooperation innerhalb netzwerkartiger Wertschöpfungssysteme genannt werden (Teece 1986; Friedkin 1993; Uzzi 1997). Als drittes wird schließlich unter dem Konstrukt Erfolg die Summe der resultierenden Effekte verstanden, die zur Erfüllung der durch den Nutzer definierten Leistungsanforderungen beitragen. Der theoretische Bezugsrahmen ist in Abb. 2 dargestellt.

Innerhalb des Bezugsrahmens stellt der Erfolg die abhängige Größe dar. Dieser setzt sich aus der Erfüllung aller funktionalen Anforderungen sowie der Einhaltung wirtschaftlicher und zeitlicher Vorgaben des Nutzers zusammen. Die Kommunikations- und Kooperationswirkungen der Wertschöpfungspartnerschaft haben dabei maßgeblichen Einfluss auf die Erfüllung dieser Leistungsanforderungen. Unter Kommunikation- und Kooperationswirkungen wird in der wirkungsanalytischen Untersuchung von Netzwerken der Wissens- und Informationsaustausch zwischen den beteiligten Organisationen zur gemeinschaftlichen Leistungserbringung verstanden (Tsai 2002, S. 179). Ausmaß und Qualität dieses Austausches wird maßgeblich von Faktoren bestimmt, die das individuelle Agieren der beteiligten Akteure beeinflussen. Die Organisationsform prägt dabei diese Faktoren und nimmt durch ihre vertragliche sowie ihre aufbau- und ablauforganisatorische Ausgestaltung Einfluss auf die Kommunikations- und Kooperationswirkungen einer Wertschöpfungspartnerschaft (Wasko und Faraj 2005, S. 40; Möller 2006, S. 184; Arya und Lin 2007, S. 701). So sorgen beispielsweise die aus der speziellen Organisationsform einer Wertschöpfungspartnerschaft resultierenden vertraglich angelegten Abhängigkeiten für starke sozioökonomische Beziehungen zwischen den Projektpartnern, wodurch ein aktives 
Kommunikations- und Kooperationsverhalten gefördert wird. Die mit der Organisationsform einer konventionellen Beschaffungsvariante verbundene Einzelvergabe kann dagegen die sozioökonomischen Beziehungen zwischen den Beteiligten belasten, da wirtschaftlich agierende Akteure in der Regel versuchen ihr Optimum aus den geschlossenen Verträgen herauszuholen und nicht versuchen kooperativ den Gesamterfolg eines Projektes zu optimieren.

Im Folgenden werden der Einfluss der Faktoren des Bezugsrahmens auf das Kommunikations- und Kooperationsverhalten der Beteiligten Akteure einer Wertschöpfungspartnerschaft dargestellt und entsprechende Wirkungszusammenhänge formuliert.

2.3 Ursachen der Kommunikations- und Kooperationswirkungen von immobilienwirtschaftlichen Wertschöpfungspartnerschaften

\subsubsection{Beziehungen}

Ein wesentlicher Einflussfaktor auf das Interaktionsverhalten zwischen Organisationen innerhalb eines Netzwerkes sind deren jeweilige Beziehungen untereinander (Uzzi 1997, S. 43 f.). Dabei beeinflusst die Stärke dieser Beziehung in welchem Umfang die Organisationen miteinander kooperieren bzw. Informationen und Wissen austauschen. Die am weitesten verbreitete Erklärung für das Ent- und Bestehen starker Beziehungen innerhalb von Netzwerken ist Vertrauen (Sydow 1992). Vertrauen zwischen Organisationen herrscht insbesondere dann, wenn bereits positive Erfahrungen miteinander bestehen (Jones und George 1998, S. 531). Durch diese gemachten Erfahrungen wissen Organisationen wie die anderen Parteien mit Informationen umgehen und wie diese bei Problemen und Konflikten reagieren; dies führt zu einer Beziehung, in der Informationen und Wissen einfacher auszutauschen sind (Larson 1992). Neben dem Aspekt der Erfahrung erwächst Vertrauen zwischen Akteuren auch aus Zielkongruenzen, wenn Akteure davon ausgehen können, dass sie aus der Weitergabe von Ressourcen an Interaktionspartner ebenfalls profitieren (Anderson und Weitz 1989, S. 18). Infolge dessen formulieren wir folgende Hypothese:

H1: Je stärker die Beziehungen zwischen Akteuren, desto häufiger tauschen diese Akteure Informationen und Wissen miteinander aus, und beeinflussen damit den Erfolg positiv.

\subsubsection{Positionierung}

Eine weitere Determinante für den Kooperationserfolg von Netzwerken ist die Netzwerkdichte, wobei unter „Netzwerkdichte“ die Anzahl direkter und indirekter Verbindungen zwischen den beteiligten Organisationen verstanden wird. Je höher diese Dichte ist, desto größer ist die Kooperationsintensität innerhalb des Netzwerkes (Gilsing et al. 2008, S. 1721). Auf Ebene der individuellen Organisation ist in diesem Kontext der Aspekt der Positionierung relevant: Je zentraler eine Organisation in einem Netzwerk positioniert ist, desto einfacher und schneller kann diese Organisation mit anderen Organisationen interagieren (Friedkin 1993, S. 864). Die höchste 
Zentralität innerhalb eines Netzwerkes besitzt die „Lead-Organisation“, eine Organisation, die aufgrund ihres Aufgabenspektrums und ihrer zentralen Positionierung ein hohes Maß an Bekanntheit und Einfluss innerhalb des Netzwerkes besitzt (Provan et al. 2007, S. 482). Lead-Organisationen tragen, durch die Etablierung formaler und informaler Kommunikationswege (Brown und Duguid 2000, S. 320) maßgeblich dazu bei, dass innerhalb eines Netzwerks Informationen und Wissen optimal ausgetauscht werden (Sydow und van Well 2010, S. 143 ff.). Infolge dessen formulieren wir folgende Hypothese:

H2: Je zentraler die Positionierung eines Akteurs (Organisation oder Individuum) innerhalb des Netzwerkes ist, desto stärker wird dieser Akteur den Erfolg beeinflussen.

\subsubsection{Motivation}

Ein wesentlicher Aspekt, der Einfluss auf die Kooperationswirkung einer Wertschöpfungspartnerschaft haben kann, sind die jeweiligen Ausprägungen der Motivation der beteiligten Organisationen hinsichtlich eines kooperativen Verhaltens. Innerhalb von Netzwerken kann der Anreiz zur Kooperation in einem ,gegenseitigen Nutzen“ bestehen, dieser kann monetärer aber auch qualitativer Natur sein (Knop 2009, S. 102). Branchenübergreifende empirische Untersuchungen zeigen, dass in Projekten mit zielgerichteten Anreizsituationen das Verhalten beteiligter Organisationen so beeinflusst werden kann, dass sich dies positiv auf den Projekterfolg ausgewirkt (Olsen et al. 2005). Dies betrifft insbesondere Aspekte wie Wirtschaftlichkeit, Pünktlichkeit und Qualität (Chua et al. 1999). In Bezug auf die Beschaffung von Immobilienprojekten ergibt sich eine spezielle Anreizsituation. Hier hat der Betreiber, aufgrund der langfristigen vertraglichen Verpflichtung das Gebäude zu einem pauschalierten Entgelt zu betreiben, den Anreiz, die Qualität des Gebäudes hinsichtlich der Betriebsphase durch Einbringung von Wissen und zusätzlichen Investitionen zu steigern (Bennet und Iossa 1972). Infolge dessen formulieren wir folgende Hypothese:

H3: Je stärker Organisationen vom kooperativen Austausch mit den anderen Organisationen profitieren können, desto intensiver werden sie sich an dem Austausch beteiligen und hierdurch den Erfolg steigern.

\subsubsection{Leistungskompetenz}

Die Qualität der Leistungserfüllung eines Akteures ist neben der Motivation insbesondere von dessen individuellen Fähigkeiten bzw. seiner Expertise abhängig. Diese sozialen und fachlichen Fähigkeiten eines Akteurs werden auch als Leistungskompetenz bezeichnet. Hierunter werden dessen fachliche Fähigkeiten, sein Fachwissen und die Erfahrungen eines Akteurs, bezüglich der geforderten Leistung, verstanden (Dolezych 2010, S. 192). Durch den gezielten Einsatz von Akteuren mit einer speziellen fachlichen Leistungskompetenz, lässt sich die Qualität einer gemeinschaftlichen Leistungserbringung optimieren (Teece 1986, S. 288). Dies betrifft insbesondere die 


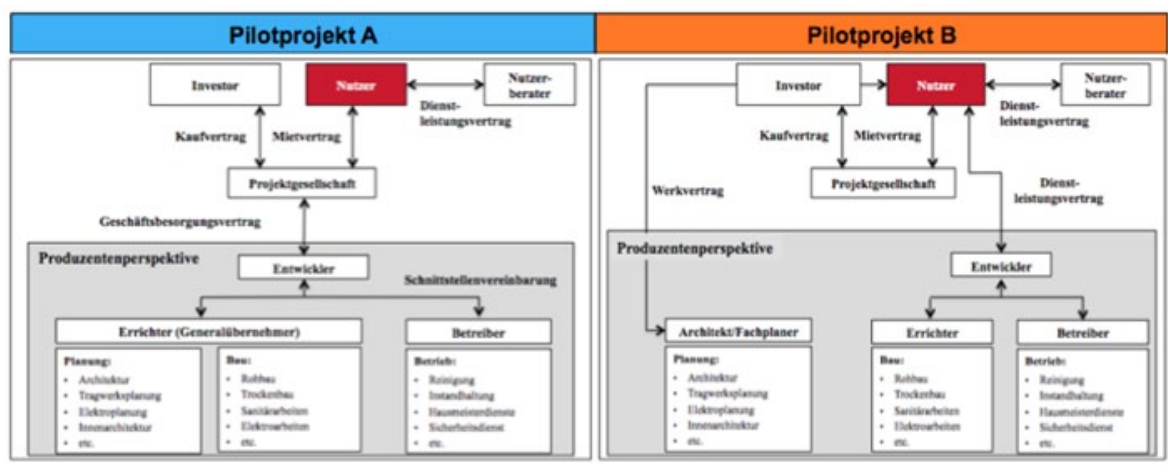

Abb. 3 Gegenüberstellung der Organisationsstruktur beider Pilotprojekte. (Eigene Darstellung)

Erbringung von Systemleistungen, (Sheperd und Ahmed 2000, S. 104) die in der Regel aus einer Vielzahl an Leistungsbestandteilen bestehen und entsprechend hohe koordinative Herausforderungen mit sich bringen. Infolge dessen formulieren wir folgende Hypothese:

H4: Je höher die Leistungskompetenz eines Akteurs hinsichtlich der Erfüllung der Leistungsanforderungen ist, desto stärker ist dessen Einfluss auf den Erfolg.

\section{Empirie}

\subsection{Untersuchungsobjekte}

Bei den beiden begleiteten Projekten handelt es sich um die ersten beiden in der Konstellation einer immobilienwirtschaftlichen Wertschöpfungspartnerschaft organisierten Projektentwicklungen nach dem Vorbild von ÖPPs in Deutschland. Es wurden jeweils die neuen Unternehmenszentralen privatwirtschaftlicher Unternehmen entwickelt. Die Immobilien haben Nutzflächen von ca. 10.000 respektive 25.000 Quadratmetern. Die Investitionsvolumina belaufen sich auf ca. 30 Mio. bzw. 85 Mio. $€$.

Beide Projekte verfolgen die Grundprinzipien einer immobilienwirtschaftlichen Wertschöpfungspartnerschaft (siehe B.I.), allerdings unterscheiden sie sich in der Zusammensetzung der beteiligten Organisationen und den entsprechenden Vertragsbeziehungen (siehe Abb. 3): In Pilotprojekt A befand sich die gesamte Verantwortung für Planung, Bau und Betrieb bei dem Entwickler. Dieser beauftragte die Bau- und Betreiberleistungen bei konzerneigenen Tochterunternehmen. Bereits während der Planungsphase wurden dann die im Nutzungsentgelt enthaltenen Betriebskosten vertraglich fixiert. Für die Architektur und Fachplanung beauftragte er externe Unternehmen, mit denen er bereits mehrere vergleichbare Projekte realisiert hatte. Der Nutzer beauftragte direkt lediglich einen Nutzerberater. In Pilotprojekt B hingegen wurden Architektur und Fachplanung von den Disziplinen Entwicklung, Bau und Betrieb bewusst durch den Nutzer vertraglich getrennt. Der Nutzer hat darüber hinaus einen Nutzerberater separat beauftragt. Eine vertragliche Einigung mit dem Betreiber über eine Kostengarantie der Betreibskosten war zum Zeitpunkt der teilnehmenden 
Anforderungskategorien

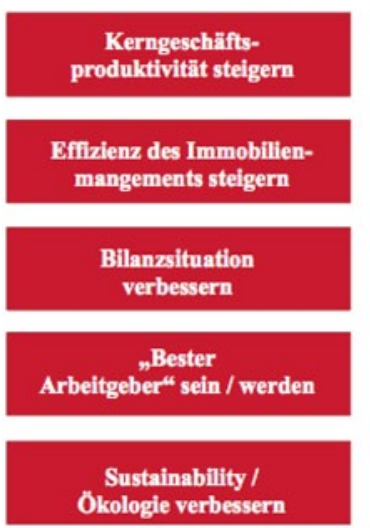

\section{Anforderungen}

\begin{tabular}{|c|c|}
\hline $\begin{array}{l}\text { - Reduzierung der Betriebskosten } \\
\text { - Reduzierung der Risiken } \\
\text { - Optimierung der Mitausgaben }\end{array}$ & $\begin{array}{l}\text { - Erhöhung der Flācheneffizienz } \\
\text { - Reduzierung der Anschaffungskosten } \\
\text { - Reduzierung der Managementkapazitäten }\end{array}$ \\
\hline $\begin{array}{l}\text { - Optimierung der Funktionsfahigkeit } \\
\text { - Steigerung der Flexibilităt } \\
\text { - Verringerung Krankheitsquote }\end{array}$ & $\begin{array}{l}\text { - Erhōhung der Zusammenarbeit-/ } \\
\text { Kommunikationseignung } \\
\text { - Eignung des Standortes }\end{array}$ \\
\hline $\begin{array}{l}\text { - Ertragskennzahlen } \\
\text { - Finanzierungskennzahlen }\end{array}$ & $\begin{array}{l}\text { - Rentabilitätskennzahlen } \\
\text { - Liquiditätskennzahlen }\end{array}$ \\
\hline
\end{tabular}

Recruitingerfolge
Mitarbeiterzufriedenheit

Work-Life-Balance

$\mathrm{CO} 2$ Reduktion

Zertifizierung
- Steigerung der Energieeffizienz

Abb. 4 Formulierte Nutzeranforderungen an die neuen Unternehmens-zentralen

Beobachtung noch nicht erfolgt. Infolge dessen gehen sämtliche Vertragsbeziehungen vom Nutzer aus. In Abb. 3 sind die Vertragsbeziehungen noch einmal vereinfacht dargestellt.

Zum Zeitpunkt der Untersuchung war der spätere Investor noch nicht bestimmt, infolge dessen ist er kein Bestandteil der Untersuchung.

\subsection{Methoden der Datenerhebung}

\subsubsection{Wissenschaftliche Begleitung durch teilnehmende Beobachtung}

Bei den jeweils wöchentlich stattfindenden Planungsbesprechungen, die teilnehmend beobachtet wurden, nahmen regelmäßig alle für die Realisierung der Projekte verantwortlichen Organisationen teil (Abb. 4). Der Beobachtungszeitraum betrug in beiden Projekten jeweils ca. 1 Jahr. Zweck der Besprechungen war es, den aktuellen Planungsstand zu besprechen und die Expertise und Anmerkungen der beteiligten Organisationen einzuholen sowie in der weiteren Planung zu berücksichtigen. Die Datenerhebung während dieser Sitzungen erfolgte mit der Methode der teilnehmenden Beobachtung (Girtler 2001). Diese Form der Datenerhebung bietet die Möglichkeit, komplexe Situationen und Handlungsprozesse beinahe unbeschränkt zu erfassen, und ist daher für die komplexe Interaktion während der Planungsbesprechungen besonders gut geeignet. Der Beobachter orientiert sich hierbei an einem Beobachtungsleitfaden. In der vorliegenden Untersuchung ergab sich der Beobachtungsleitfaden aus den formulierten Anforderungen der Nutzer an die Beschaffung der Immobilien. Aus fünf Anforderungskategorien wurden insgesamt 22 Anforderungen formuliert.

Die Beobachtungen wurden in Form von Beobachtungsprotokollen festgehalten. 


\subsubsection{Erhebung der internen Einflussfaktoren des theoretischen Bezugsrahmens}

Zur Erhebung der internen Einflussfaktoren „Beziehungen“, „Motivation“ und „Expertise“ (Abb. 2) wurden mit allen beteiligten Akteuren problemzentrierte Interviews geführt. Zweck der Interviews war es, für jeden Akteur die jeweiligen Ausprägungen der internen Einflussfaktoren zu erheben. Hierfür wurden die Interviewten gebeten eine paarweise vergleichende Bewertung der Ausprägungen der einzelnen Einflussfaktoren für jede mögliche Paarung der beteiligten Organisationen vorzunehmen. Die Bewertung erfolgte anhand einer 9-stufigen Skala ( 1 = gleichwertig, $9=$ extrem höher). Hierzu wurden für die Einflussfaktoren folgende Indikatoren definiert:

Für den Einflussfaktor „,Beziehungen “ wurde als Indikator das Vertrauensverhältnis zwischen den Organisationen gewählt. Entsprechend wurde jeder Akteur in den geführten Interviews direkt nach dem Vertrauensverhältnis ihrer Organisation zu den jeweils beteiligten Organisationen und nach den Vertrauensverhältnissen der anderen Organisationen untereinander befragt.

Für den Einflussfaktor „Leistungskompetenz “ wurde als Indikator die Kernkompetenz der beteiligten Organisationen zugrunde gelegt. Als Kernkompetenz wurden die Fähigkeiten der beteiligten Organisation hinsichtlich der definierten Leistungsanforderungen des Nutzers bewertet (Prahalad und Hamel 1990, S. 83).

Hinsichtlich des Einflussfaktors „,Motivation“ wurde die extrinsische instrumentelle Motivation untersucht. Hierbei handelt es sich um eine Form der Motivation, bei der das Verhalten eines Menschen von der Aussicht auf einen konkreten Vorteil geleitet wird (Barbuto und Scholl 1998, S. 1011). Als Indikator dient der wirtschaftliche Anreiz, den die Organisationen hinsichtlich einer wirtschaftlich effizienten und qualitativ hochwertigen Leistungserbringung in den beiden begleiteten Pilotprojekten haben.

Für den Einflussfaktor „, Positionierung “wurde als Indikator die Anzahl der Interaktionen einer Organisation mit der Lead-Organisation des Netzwerkes bestimmt. Hierzu wurde im Netzwerk bzw. in der Wertschöpfungspartnerschaft zuerst die LeadOrganisation bestimmt. Hierfür wurde die Maßgröße der Degree-Zentralität verwendet, die ausdrückt, wie viele direkte Verbindungen eine Organisation innerhalb eines Netzwerkes auf sich vereinigt (Wasserman und Faust 1994, S. 178).

\subsection{Methoden der Datenanalyse}

\subsubsection{Qualitative Inhaltsanalyse zur Auswertung der Beobachtungsprotokolle}

Die Auswertung der Beobachtungsprotokolle hinsichtlich der koordinativen Wirkung der beiden immobilienwirtschaftlichen Wertschöpfungspartnerschaften erfolgte mit Hilfe einer qualitativen Inhaltsanalyse. Hierbei handelt es sich um eine spezielle Form der Analyse, bei der durch ein systematisches, regelgeleitetes Vorgehen sprachliches Material in protokollierter Form analytisch zerlegt und interpretiert wird (Mayring 2002). Im Zentrum jeder qualitativen Inhaltsanalyse steht dabei ein Kategoriensystem. In der vorliegenden Untersuchung entstand das Kategoriensystem aus den 21 Anforderungen der beiden Nutzer. Es bildet den Maßstab der „Funktionserfüllung“ im Erfolgs-Konstrukt der Untersuchung. Mit Hilfe dieses Kategoriensystems 


\begin{tabular}{|l|l|}
\hline Nutzer-Anforderung & Beispiel für einen Effekt \\
\hline Reduzierung der & $\begin{array}{l}\text { "Betreiber schlägt für die Decke in der Küche anstatt wie geplant } \\
\text { Gipskarton eine Blechklemmdecke vor, da diese viel einfacher und besser } \\
\text { zu reinigen ist. }\end{array}$ \\
\hline $\begin{array}{l}\text { Erhöhung der } \\
\text { Funktionsfähigkeit }\end{array}$ & $\begin{array}{l}\text { "Betreiber macht Anmerkungen über die Positionierung der } \\
\text { Revionsklappen in den Mittelzonen; bei jetzigem Stand der Planung ist }\end{array}$ \\
\hline Steigerung der & $\begin{array}{l}\text { "Fachplaner macht Anmerkungen zur geplanten Arbeitsplatzgestaltung } \\
\text { und liefert einen Vorschlag hinsichtlich des Mobiliars und den } \\
\text { Materialien, wodurch die lokalen akustischen Verhältnisse für den } \\
\text { jeweiligen Mitarbeiter verbessert werden können. “ }\end{array}$ \\
\hline Reduzierung der Risiken & $\begin{array}{l}\text { "Entwickler, Nutzerberater und Betreiber besprechen gemeinsam die } \\
\text { Planung und Gestaltung von Sonderflächen damit diese den } \\
\text { Anforderungen des Brandschutze entsprechen. “ }\end{array}$ \\
\hline
\end{tabular}

Abb. 5 Beispiele für beobachtete Effekte

wurden in den Beobachtungsprotokollen die entsprechenden Textstellen (Codings) extrahiert, in denen die Anforderungen des Nutzers durch kooperatives Verhalten (Austausch von Informationen und Wissen) der beteiligten Organisationen positiv beeinflusst wurden (Mayring 2000, S. 92). Jedes dieser qualitätssteigernden Ereignisse, bei dem durch aktiven Informations- und Wissenseintrag einer oder mehrerer der beteiligten Organisationen das jeweilige Projekt hinsichtlich der Anforderungen der Nutzer über das ursprüngliche Planungsniveau hinaus positiv beeinflusst wurde, wird als „Effekt" bezeichnet. Für eine differenzierende Bearbeitung wurden für jeden Effekt die beteiligten Organisationen vermerkt. So war es anschließend möglich, die beobachteten Effekte den verursachenden Organisationen zuzuordnen. In Abb. 5 sind für ausgewählte Nutzeranforderungen beispielhafte Effekte aufgeführt.

Zur Objektivierung der Ergebnisse wurde die qualitative Inhaltsanalyse der Protokolle von drei unabhängigen Bearbeitern durchgeführt. Als Gütekriterium wurde die Intercoder-Reliabilität gewählt. Diese gibt den Grad der Übereinstimmung, der an einem Text vorgenommenen Kodierungen, von mindestens zwei unabhängigen Kodierern wieder (Mayring 2000, S. 110). Der entsprechende Reliabilitätskoeffizient (Übereinstimmung der Codings) der Untersuchung lag bei $81 \%$, so dass die folgenden Ergebnisse als belastbar eingestuft werden können (Krippendorf 1980, S. 133).

\subsubsection{Analyse der formulierten Wirkungszusammenhänge}

Zur Überprüfung der in den Hypothesen formulierten Wirkungszusammenhänge, wurden wie bereits beschrieben, für jede der beteiligten Organisationen die Ausprägungsstärken der, zur Operationalisierung der internen Einflussfaktoren eingeführten Indikatoren erhoben. Anschließend wurden für jeden Indikator die jeweiligen Ausprägungsstärken der beteiligten Organisationen mittels der Methode des Analyitical Hierarchy Process (AHP) in eine hierarchische Rangfolge gebracht. Hierbei wur- 


\begin{tabular}{|c|c|c|c|c|c|c|c|c|c|c|}
\hline & \multicolumn{4}{|c|}{ Evaluationsmatrix } & \multicolumn{4}{|c|}{ Normalisierung } & \multirow[b]{2}{*}{$r_{i}$} & \multirow{2}{*}{$\begin{array}{c}\text { Gewicht } \\
w\end{array}$} \\
\hline & $a_{1}$ & $a_{2}$ & $\ldots$ & $a_{n}$ & $a_{1}$ & $a_{2}$ & $\ldots$ & $a_{n}$ & & \\
\hline$a_{1}$ & $a_{11}=1$ & $a_{12}$ & $\cdots$ & $a_{1 n}$ & $a_{11} / c_{1}$ & $a_{12} / c_{2}$ & $\ldots$ & $a_{1 n} / c_{n}$ & $r_{1}$ & $w_{1}=r_{1} / n$ \\
\hline$a_{2}$ & $a_{21}=1 / a_{12}$ & 1 & & $a_{2 n}$ & $a_{21} / c_{1}$ & $a_{22} / c_{2}$ & $\cdots$ & $a_{2 n} / c_{n}$ & $r_{2}$ & $w_{2}=r_{2} / n$ \\
\hline$\vdots$ & & $\vdots$ & & $\vdots$ & $\vdots$ & $\vdots$ & & $\vdots$ & $\vdots$ & $\vdots$ \\
\hline$a_{n}$ & $a_{n 1}=1 / a_{1 n}$ & $a_{2 n}$ & $\cdots$ & $a_{n n}=1$ & $a_{n 1} / c_{1}$ & $a_{n 2} / c_{2}$ & $\cdots$ & $a_{n n} / c_{n}$ & $r_{n}$ & $w_{n}=r_{n} / n$ \\
\hline$c_{i}$ & $c_{1}=\sum_{i=1}^{n} a_{i 1}$ & $c_{2}=\sum_{i=1}^{n} a_{i 2}$ & $\ldots$ & $c_{n}$ & 1 & 1 & $\cdots$ & 1 & $n$ & 1 \\
\hline
\end{tabular}

Abb. 6 Hierarchisierung der Indikatorenausprägung mit der AHP-Verfahren. (Meixner und Haas 2002)

den die jeweiligen Ausprägungen der Indikatoren Vertrauen, wirtschaftlicher Anreiz und Leistungskompetenz der beteiligten Personen paarweise gegenübergestellt und jeweils von jedem Projektakteur unabhängig bewertet. Für diese vergleichende Bewertung wurde eine Skala mit einer Bandbreite von 1 bis 9 Punkten $(1=$ gleichwertig, 9=extrem höher) verwendet. Der paarweise Vergleich erfolgte für jeden Indikator unabhängig und jeweils in einer eigenen Evaluationsmatrix. Hierbei repräsentiert $\mathrm{a}_{\mathrm{i}}$ die beteiligten Organisationen und $\mathrm{a}_{\mathrm{ij}}(1 \leq \mathrm{i} \leq \mathrm{n}, 1 \leq \mathrm{j} \leq \mathrm{n})$ die resultierenden Paarvergleiche (Abb. 6).

Um die Paarvergleichswerte verknüpfen zu können, wurden diese auf eine vergleichbare Basis gebracht. Dazu wurden die Spaltensummen $\left(\mathrm{c}_{\mathrm{i}}\right)$ der Evaluationsmatrix bestimmt. Anschließend wurde diese auf 1 normiert. Danach wurde aus der normalisierten Matrix die Zeilensumme $\left(\mathrm{r}_{\mathrm{i}}\right)$ gebildet und durch die Anzahl der Elemente dividiert, womit sich das Gewicht w für das jeweilige Element ergab. Aus den unterschiedlichen Gewichten ergibt sich die Hierarchisierung der Indikatorenausprägungen nach den beteiligten Organisationen. So ergibt sich für jeden Indikator eine Verhältnisskala, auf der jeder beteiligten Organisation ein Rang bzw. ein Wert hinsichtlich der jeweiligen Indikatorenausprägung zugeordnet werden konnte (Saaty 1986). In einem nächsten Schritt wurden für jede Organisation die jeweiligen Werte der Indikatorenausprägungen mit der Anzahl der beobachteten Effekte dieser Organisation kombiniert und hinsichtlich eines potentiellen Zusammenhangs von Indikatorenausprägung und Wissens- und Informationsaustausch analysiert.

Diese Wirkungsanalyse wurde mittels einer Korrelationsanalyse durchgeführt. Aufgrund der relativ geringen Datenmenge wurde auf komplexere statistische Analyseverfahren, wie beispielsweise eine Regressionsanalyse verzichtet.

\section{Ergebnisse}

4.1 Erste univariate Auswertungen zu den Erfolgswirkungen immobilienwirtschaftlicher Wertschöpfungspartnerschaften

Durch die qualitative Inhaltsanalyse der Beobachtungsprotokolle des Pilotprojektes A konnten insgesamt 109 optimierende Effekte und aus den Protokollen des Pilotprojektes B 85 dieser Effekte extrahiert werden. In Abb. 7 ist die prozentuale Verteilung 


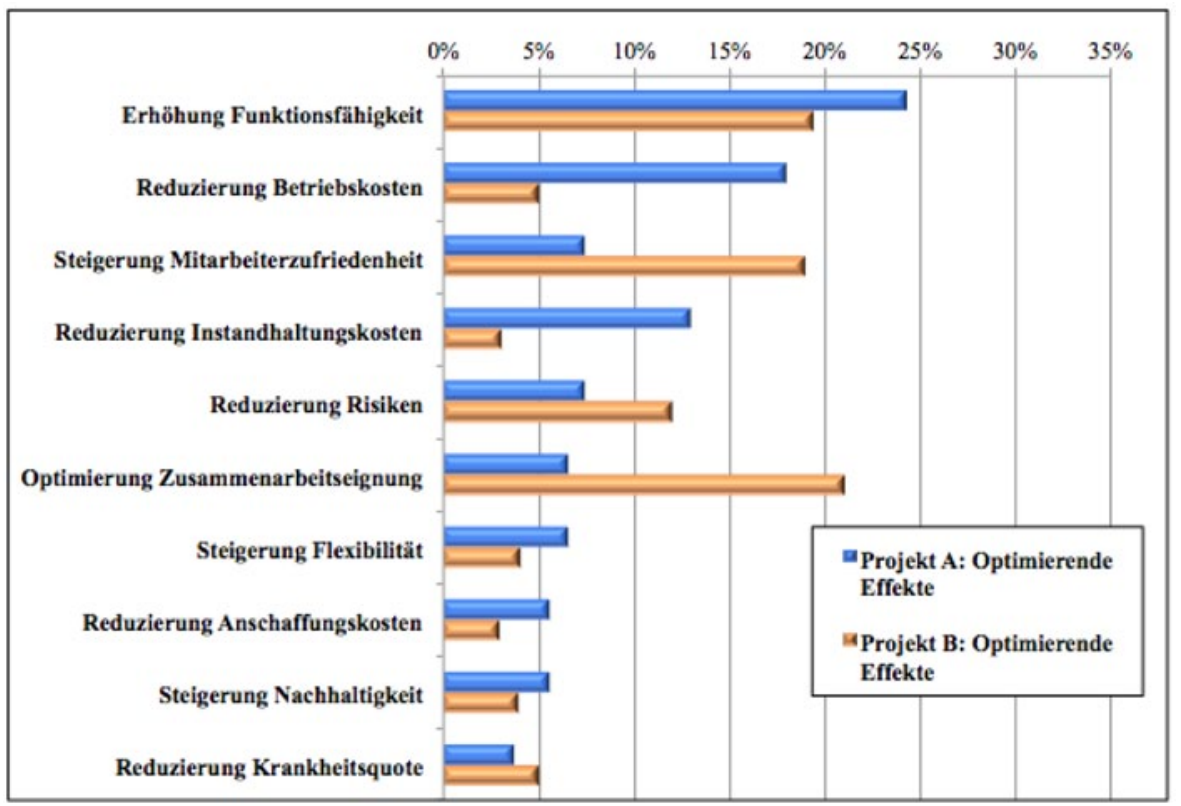

Abb. 7 Prozentuale Verteilung der beobachteten Effekte beider Erfahrungsobjekte

der Effekte beider Pilotprojekte für die am häufigsten beeinflussten Nutzeranforderungen dargestellt.

Es zeigt sich, dass in beiden Pilotprojekten die Anforderung ,,Erhöhung der Funktionsfähigkeit " durch die kooperative Zusammenarbeit der beteiligten Organisationen prozentual am häufigsten positiv beeinflusst wurde. In Pilotprojekt A wurde am zweithäufigsten die Anforderung „,Reduzierung der Betriebskosten“ optimiert. Die Effekte zur Reduzierung der Betriebskosten betrafen in der Mehrheit Entscheidungen bezüglich der zu verwendenden Materialien und Systeme, infolge derer sich zum Beispiel die Reinigungs- und Wartungskosten reduzieren ließen. Demgegenüber belegen in Pilotprojekt B die Effekte hinsichtlich der Anforderung ,, Optimierung der Zusammenarbeitseignung “ Rang zwei.

Für beide Projekte kann nach Auswertung der Beobachtungsprotokolle festgehalten werden, dass es durch den integralen Wissens- und Informationsaustausch der beteiligten Organisationen, gegenüber den ursprünglichen Planungen, zu einer deutlichen Erhöhung der einzelnen Qualitäten und damit des angestrebten Erfolges aus Nutzersicht kam. Dabei lag in Pilotprojekt A der Fokus der Effekte insbesondere auf der Erfüllung wirtschaftlicher und in Pilotprojekt B auf der Erfüllung qualitativer Anforderungen.

\subsection{Wirkungsanalytische Untersuchung des Kooperationserfolges}

Zur Untersuchung inwieweit die Einflussfaktoren des theoretischen Bezugsrahmens das Interaktionsverhalten der beteiligten Akteure beeinflusst haben, wurden die beobachteten Effekte zunächst nach den verursachenden Akteuren geordnet. In Abb. 8 ist 




Abb. 8 Prozentuale Verteilung der Beteiligungen der Organisationen an den beobachteten Effekte

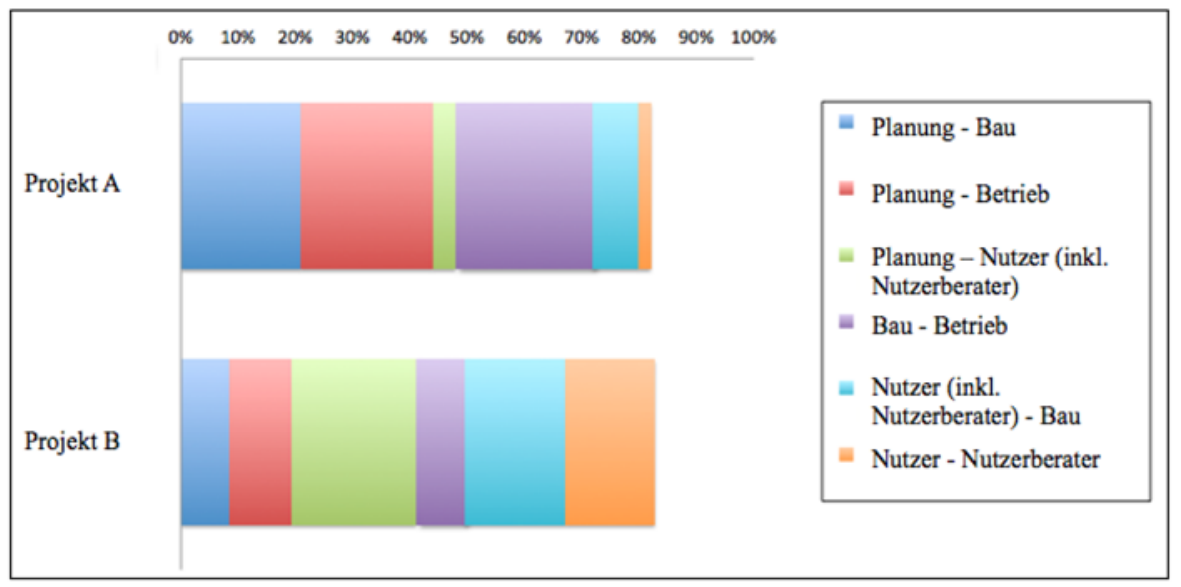

Abb. 9 Prozentuale Verteilung gemeinschaftlicher Effekte auf die beteiligten Wertschöpfungsstufen

die Verteilung der Effekte auf die verursachenden bzw. an der Entstehung beteiligten Organisationen dargestellt. Wie aus der Abbildung ersichtlich wird, gestaltet sich die Verteilung der Effekte auf die jeweils verantwortlichen Organisationen in beiden Projekten deutlich unterschiedlich.

In Pilotprojekt A sind maßgeblich die Organisationen der Wertschöpfungsstufen Planung, Bau und Betrieb für die beobachteten Effekte verantwortlich. Insbesondere dem Betreiber konnten 37\% der Effekte, und damit der mit Abstand größte Einzelanteil zugeschrieben werden. Die Organisationen der Nutzerperspektive (Nutzer und Nutzerberater) haben dagegen nur einen relativ kleinen Anteil an den beobachteten Effekten. In Pilotprojekt B hingegen, sind die Anteile an den beobachteten Effekten deutlich homogener verteilt. Darüber hinaus sind Organisationen der Nutzerpers- 


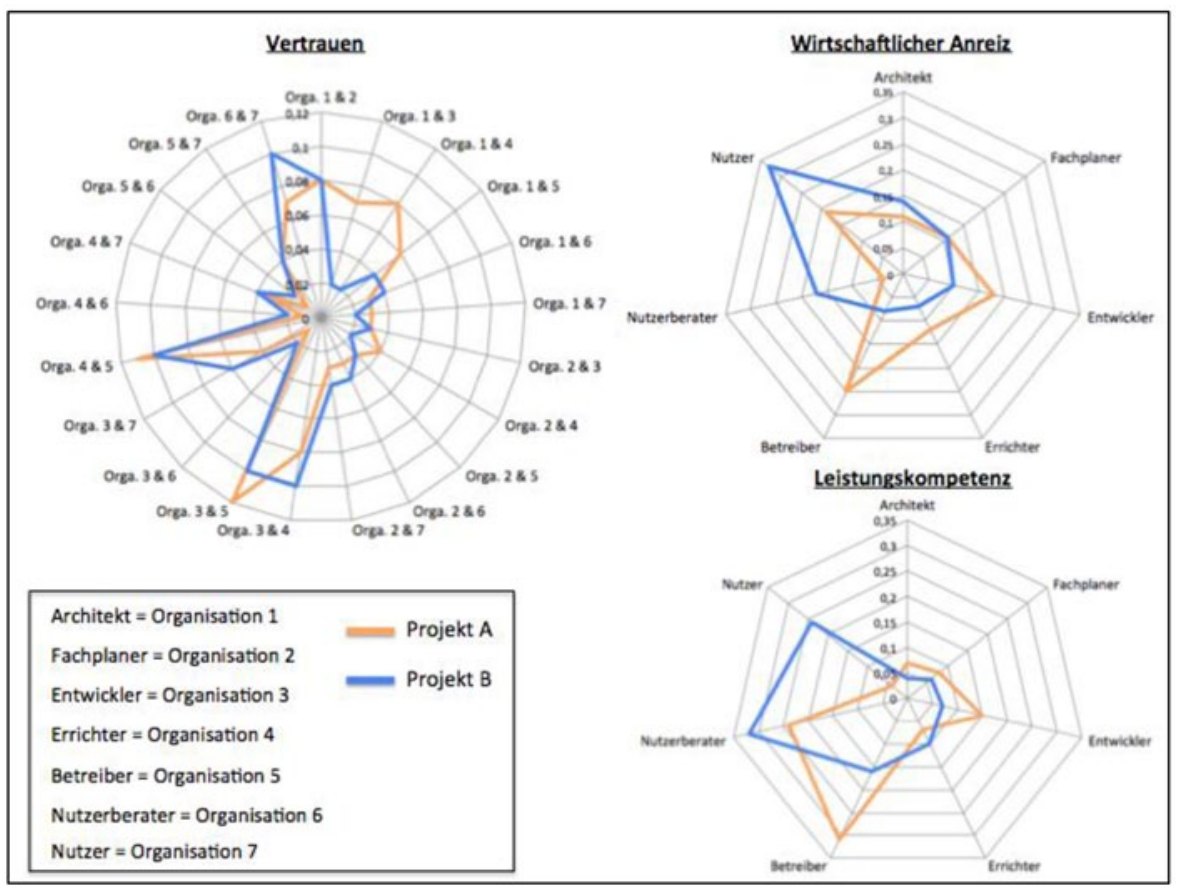

Abb. 10 Gegenüberstellung der Ausprägung der Indikatoren Vertrauen, wirtschaftlicher Anreiz und Leistungskompetenz nach Organisationen

pektive im Gegensatz zu Pilotprojekt A für rund 35\% aller beobachteten Effekte verantwortlich.

Auch hinsichtlich der Zusammensetzung der gemeinschaftlich verursachten Effekte von zwei oder mehreren Organisationen zeigen sich in beiden Pilotprojekten deutliche Unterschiede (Abb. 9) So entfallen in Pilotprojekt A auf die Organisationen der Wertschöpfungsstufen Planung, Bau und Betrieb über $40 \%$ der durch mehrere Organisationen gemeinsam verursachten Effekte. Dies zeigt einen intensiven Wissens- und Informationsaustausch zwischen den für die Planung, den Bau und den Betrieb verantwortlichen Akteuren. In Pilotprojekt B hingegen beträgt dieser Anteil lediglich ca. $18 \%$. Aus diesem Verhältnis geht hervor, dass der gemeinsame Wissenstransfer zwischen den Wertschöpfungsstufen Planung und Bau in Pilotprojekt B deutlich geringer ausfällt. Hingegen fand in Pilotprojekt B mit rund $23 \%$ vor allem ein intensiver Austausch zwischen den Organisationen der Planung und dem Nutzer und zwischen dem Nutzer und dem Nutzerberater statt.

\subsubsection{Hierarchisierung der Indikatorenausprägungen der internen Einflussfaktoren}

Für die Überprüfung der in den Hypothesen formulierten Wirkungszusammenhänge, wurden in diesem Schritt die Indikatorenausprägungen der vier internen Einflussfaktoren für jede der beteiligten Organisationen mit der AHP-Methode ausgewertet. Wie bereits in der Erläuterung der AHP-Methode beschrieben, wurden die ermittel- 
Abb. 11 Zentralität der beteiligten Organisationen

\begin{tabular}{|l|c|l|c|}
\hline \multicolumn{2}{|c|}{ Pilotprojekt A } & \multicolumn{2}{c|}{ Pilotprojekt B } \\
\hline & Zentralität & & Zentralität \\
\hline Betreiber & 0,36 & Nutzerberater & 0,44 \\
\hline Errichter & 0,25 & Architekt & 0,19 \\
\hline Architekt & 0,16 & Fachplaner & 0,16 \\
\hline Fachplaner & 0,12 & Errichter & 0,09 \\
\hline Nutzerberater & 0,08 & Entwickler & 0,08 \\
\hline Nutzer & 0,03 & Betreiber & 0,04 \\
\hline
\end{tabular}

ten Ausprägungen der Indikatoren jeweils auf einen Wert von 1 normiert (siehe III. b.), somit liegen die jeweiligen Werte zwischen 0 und 1. In Abb. 10 sind für beide Projekte die Ergebnisse des Hierarchisierungsprozesses der Indikatoren Vertrauen, wirtschaftlicher Anreiz und Leistungskompetenz dargestellt.

Wie in der Abbildung zu erkennen ist, wurden die Indikatoren der Einflussfaktoren in beiden Projekten deutlich unterschiedlich von den beteiligten Akteuren beurteilt. So geht aus der Analyse unter anderem hervor, dass sich in den beiden Pilotprojekten insbesondere die Stärken der Vertrauensbeziehungen zwischen den beteiligten Organisationen deutlich unterscheiden. Der größte Unterschied befindet sich demnach zwischen den Vertretern der Wertschöpfungsstufen Planung und Bau. So wurde im Pilotprojekt A das Vertrauen zwischen dem Architekten und dem Entwickler beziehungsweise dem Errichter als besonders ausgeprägt bewertet. Hier wurden der Architekt und der Errichter direkt vom Entwickler beauftragt. Im Gegensatz hierzu wurden in Pilotprojekt B der Architekt, der Errichter und der Entwickler separat vom Nutzer beauftragt, somit existierte keine vertragliche Verbindung zwischen den Wertschöpfungsstufen Planung und Bau. Als vermeintliche Konsequenz wurden die Vertrauensverhältnisse im Pilotprojekt B zwischen diesen beiden Wertschöpfungsstufen als wenig ausgeprägt bewertet.

Bezüglich des wirtschaftlichen Anreizes beurteilen die Projektbeteiligten, dass aufgrund der vertraglichen Konstellationen der Betreiber, welcher in Pilotprojekt A die Betriebskosten der ersten 15 Jahre garantiert, das größte Interesse daran hat, die planerische und bauliche Qualität des Projektes zu optimieren. In Pilotprojekt B hingegen wird der größte wirtschaftliche Anreiz dem Nutzer zu gesprochen, der in der begleiteten Projektphase die gesamte wirtschaftliche Verantwortung des Projektes getragen hat. Hinsichtlich des Indikators der Leistungskompetenz besteht der größte Unterschied in der Bewertung des Nutzers. In Pilotprojekt A wurde der Nutzer durch einen Vertreter der Geschäftsführung in den wöchentlichen Planungsbesprechungen vertreten, dieser hatte bis dato keine Expertise hinsichtlich der Planung und Realisierung von Immobilienprojekten. In Pilotprojekt B hingegen wurde die wöchentliche Planungsbesprechung vom Leiter des CREM geleitet, welcher über eine entsprechende Expertise verfügte.

Hinsichtlich des internen Einflussfaktors „Positionierung“ hat die Berechnung der Degree-Zentralität ergeben, dass in Pilotprojekt A der Entwickler und in Pilotprojekt B der Nutzer die Lead-Organisationen innerhalb der Wertschöpfungspartnerschaft darstellen. Aus der Anzahl der Interaktionen mit diesen beiden Organisationen und 


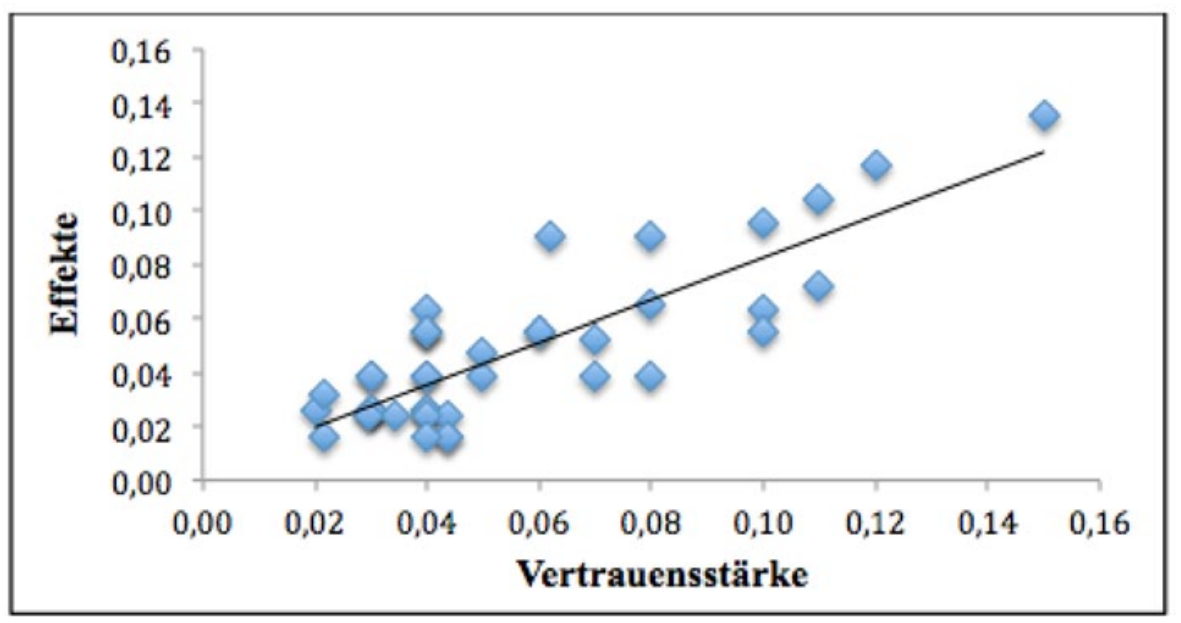

Abb. 12 Zusammenhang von Vertrauen und gemeinsamen Informations- und Wissensaustausches für Pilotprojekt A und B

der Durchführung des AHP-Verfahrens ergeben sich für beide Projekte die folgenden Rangfolgen der Zentralität der beteiligten Organisationen (Abb. 11).

\subsubsection{Zusammenhang zwischen internen Einflussfaktoren und kooperativer Wirkung immobilienwirtschaftlicher Wertschöpfungspartnerschaften}

Hypothese 1 prognostiziert, dass, je größer das Vertrauen zwischen zwei oder mehreren Organisationen ist, desto häufiger werden Informationen und Wissen miteinander ausgetauscht und desto mehr erfolgswirksame Effekte werden generiert. In Abb. 12 ist der Zusammenhang zwischen der Stärke der Vertrauensbeziehungen und den von mehreren Organisationen gemeinsam verursachten Effekten für beide Pilotprojekte dargestellt. Aus der Abbildung geht deutlich hervor, dass die Ausprägung der Vetrauensstärke zwischen zwei Organisationen Einfluss darauf hat, wie intensiv bzw. häufig diese Informationen und Wissen im Rahmen einer gemeinschaftlichen Leistungserbringung austauschen. Die durchgeführte Korrelationsanalyse bestätigt die statistische Signifikanz dieses Zusammenhangs $(\mathrm{r}=0,838 ; p<0,01)$, somit kann die in Hypothese 1 aufgestellte Vermutung im Rahmen dieser Untersuchung bestätigt werden.

Dagegen konnte der in Hypothese 2 prognostizierte Zusammenhang, dass das Ausmaß des Informations- und Wissenseintrages einer Organisation zur Optimierung des Projekterfolges mit der Zentralität ihrer Positionierung innerhalb eines Netzwerkes korreliert, infolge der durchgeführten Korrelationsanalyse nicht bestätigt werden ( $\mathrm{r}=0,138$; n. s.).

In Hypothese 3 wurde prognostiziert, dass, je stärker Organisationen vom kooperativen Austausch mit anderen Organisationen innerhalb eines Netzwerkes profitieren, sie sich desto mehr am Austausch von Wissen und Informationen beteiligen und so versuchen, den Erfolg des gesamten Projektes zu steigern. Die Ergebnisse der Korrelationsanalyse zeigen einen deutlichen positiven signifikanten Zusammenhang 
$(\mathrm{r}=0,701 ; p<0,05)$ zwischen dem wirtschaftlichen Anreiz und dem Anteil an den beigetragenen Effekten der jeweiligen Organisationen. Dieser statistische Nachweis bestätigt die Resultate der deskriptiven Auswertungen, die für Pilotprojekt A zeigten, dass insbesondere der Betreiber, der die betriebsbezogenen Leistungen für mindestens 15 Jahre garantiert, für die meisten Effekte verantwortlich war.

Der in Hypothese 4 prognostizierte Zusammenhang von Wissens- und Informationseintrag einer Organisation und dem Ausmaß der relevanten Expertise bzw. der Leistungskompetenz konnte im Rahmen der durchgeführten Korrelationsanalyse nicht als signifikant bestätigt werden ( $r=0,081$; $n$. $s$.).

Die Ergebnisse der Korrelationsanalyse zeigen, dass insbesondere die Einflussfaktoren Vertrauen und Motivation maßgeblich Einfluss darauf nehmen, wie intensiv Organisation versuchen durch gezielten Eintrag und Verteilung von Wissen und Informationen das Ergebnis eines Projektes zu optimieren.

\section{Diskussion und Interpretation der Ergebnisse}

Bei den beiden als Wertschöpfungspartnerschaft ausgestalteten Pilotprojekten konnte während der jeweiligen Planungsbesprechungen ein aktiver Informations- und Wissenstransfer zwischen den beteiligten Organisationen beobachtet werden, wodurch sich eine Qualitätssteigerung für beide Nutzer ergab. Diese positiven Effekte wären in einer konventionellen Beschaffungsvariante aller Voraussicht nach aufgrund prozessualer und aufbauorganisatorischer Barrieren oder mangelnder Anreize so nicht aufgetreten.

In Anbetracht der theoretischen Vorüberlegungen überrascht es nicht, dass die Aspekte Vertrauen und Motivation positive Einflüsse auf die Bereitschaft der Organisationen haben, Informationen und Wissen zur Optimierung des Projekterfolges auszutauschen. Auffallend sind allerdings die unterschiedlichen Ausprägungen dieser Einflussfaktoren in beiden Projekten. Die Ursache für die unterschiedlichen Vertrauensverhältnisse und den entsprechend ungleichverteilten Effekte liegt nach Auswertung der problemzentrierten Interviews vor allem in den abweichenden vertraglichen Strukturen innerhalb der Projekte. So führt die vertragliche Separation von Planung und Bau in Projekt B zu Zielkonflikten und Informationsasymmetrien zwischen den Organisationen. Diese rühren daher, dass die Organisationen der Planung (Architekt und Fachplaner) die Anforderungen des beauftragenden Nutzers aus ihrer Sicht bestmöglich planerisch umsetzen wollen, während die Organisationen der Wertschöpfungsstufen Bau und Betrieb aufgrund ihrer Übernahme der Investitionsund Betriebsrisiken bedacht sind, die Risikopotentiale zu reduzieren, um ihren eigenen zukünftigen wirtschaftlichen Erfolg nicht zu gefährden.

Aufgrund dieser Zielkonflikte und der entsprechend belasteten sozialen Beziehungen, resultieren in den Projekten unterschiedlich starke Kooperationsintensitäten zwischen den beteiligten Akteuren, die einen jeweils anderen Optimierungsfokus zur Folge haben. So wurden in Projekt A verstärkt optimierende Effekte hinsichtlich wirtschaftlich orientierter Nutzeranforderungen beobachtet. Auch Studien belegen, dass für eine wirtschaftlich erfolgreiche Realisierung von Immobilienprojekten eine enge Kooperation und Abstimmung zwischen den Wertschöpfungsstufen Planung und 
Ausführung erforderlich ist (Evbuomwana und Anumbab 1998; Love et al. 1998; Uhl und Höppner 2010). Die Ursache hierfür liegt in der moderierenden Wirkung der spezifischen Leistungskompetenzen der Akteure dieser Wertschöpfungsstufen, wodurch die Kooperationswirkung dieser Organisationen hinsichtlich der Wirtschaftlichkeit eines Projektes verstärkt wird. Darüber hinaus ist die Fokussierung auf eine wirtschaftliche Optimierung des Projektes auch auf die vertraglich geregelte Garantie der Betriebskosten durch den Betreiber zurückzuführen, was auch die hohe Anzahl der registrierten Effekte durch den Betreiber erklärt.

In Projekt B hingegen bestand eine starke Kooperationsintensität zwischen den Akteuren der Planung und den Nutzervertretern. Hierdurch lag der Optimierungsfokus nicht vornehmlich auf wirtschaftlichen Aspekten, sondern infolge der Integration nutzerspezifischer Leistungskompetenzen vor allem auf den qualitativen Anforderungen des Nutzers.

Um allen möglichen Missverständnissen vorzubeugen, ist aus methodischer Sicht zunächst darauf hinzuweisen, dass es sich hier um zwei Fallstudien handelt, aus denen im inferenzstatistischen Verständnis von externer Validität eigentlich nicht auf andere Fälle geschlossen werden kann. Im Sinne qualitativ theoriebildender Argumentation bilden die Ergebnisse der Fallstudie aber sehr wohl Ankerpunkte für Hypothesen, die in folgenden Analysen auf ihre Allgemeingültigkeit getestet werden können. In diesem Sinne lässt sich aus den gewonnen Erkenntnissen für das Real Estate Management vermuten, dass es durch den lebenszyklusübergreifenden Informations- und Wissensaustausch einer Wertschöpfungspartnerschaft die Qualität eines Projektes auch in anderen als den hier untersuchten Situationen gegenüber einer konventionellen Beschaffungsvariante deutlich erhöhen kann. Die Ergebnisse verdeutlichen aber auch, dass Projekte, die als Wertschöpfungspartnerschaften realisiert werden und dabei eine annähernd gleiche organisatorische und prozessuale Ausgestaltung aufweisen, deutlich unterschiedliche Ergebnisse hervorbringen können. Für eine zielgerichtete und entsprechend effektive Anwendung dieser innovativen Beschaffungsvariante ist daher festzuhalten, dass nicht das eine Idealmodell einer Wertschöpfungspartnerschaft existiert, sondern dass im konzeptionellen Design der Projekte ein konfigurativer Ansatz (Miller 1981; Miller und Mintzberg 1983) verfolgt werden sollte. Hierbei ist das Verständnis über den Einfluss sozialer Beziehungen auf das Kooperationsverhalten der beteiligten Akteure wesentlich. Die Konfiguration des konzeptionellen Designs einer Wertschöpfungspartnerschaft sollte sich daher nach dem Bauherrentyp bzw. Anforderungsprofil des Nutzers richten, so dass vor allem diejenigen Akteure intensiv kooperieren, die hinsichtlich des Anforderungsprofils die höchste Leistungskompetenz besitzen. So sollten beispielsweise Nutzer mit einem wirtschaftlich geprägten Anforderungsprofil in der Ausgestaltung der Vertragsbeziehungen insbesondere auf die Etablierung von Zielkomplementaritäten zwischen den Akteuren der Planung und der Ausführung achten.

Open Access Dieser Artikel unterliegt den Bedingungen der Creative Commons Attribution License. Dadurch sind die Nutzung, Verteilung, und Reproduktion erlaubt, sofern der/die Originalautor/en und die Quelle angegeben sind. 


\section{Literatur}

Anderson, E., \& Weitz, B. (1989). The use of pledges to build and sustain commitment in distribution channels. Journal of Marketing Research, 29, 18-34.

Arya, B., \& Lin Z. (2007). Understanding collaboration outcomes from an extended resource-based view perspective: The roles of organizational characteristics, partner attributes, and network structures?. Journal of Management, 33, 697-723.

Barbuto, J. E., \& Scholl, R. W. (1998). Motivation sources inventory: development and validation of new scales to measure an integrative taxonomy of motivation. Psychological Reports, 82, 1011-1022.

Bennet, J., \& Iossa, E. (2006). Building and managing facilities for public services. Journal of Public Economics, 90(10), 2143-2160.

Brown, J. S., \& Duguid, P. (2000). The social life of information. Cambridge: Harvard Business School Press.

Budäus, D., \& Grüb, B. (2007). Public Private Partnership: Theoretische Bezüge und praktische Strukturierung. Zeitschrift für öffentliche und gemeinwirtschaftliche Unternehmen, 30(3), 245-272.

Bundesrechnungshof. (2011). Gemeinsamer Erfahrungsbericht zur Wirtschaftlichkeit von ÖPP-Projekten, Wiesbaden: ohne Verlag.

Chua, D. K. H., Kog, Y. C., \& Loh, P. K. (1999), Critical success factors for different project objectives. Journal of Construction Engineering and Management, 125, 142-150.

Dolezych, T. (2010). Determinanten der Beziehungsqualität im B \& B Marketing. In: D. Woisetschläger, M. Michaelis, H. Evanschitzky, A. Eiting, \& C. Backhaus (Hrsg.), Marketing von Solutions, Wiesbaden: Gabler.

Evbuomwan, N. F. O., \& Anumba, C. J. (1998). An integrated framework for concurrent life-cycle design and construction. Advances in Engineering Software, 29, 587-597.

Friedkin, N. E. (1993). Structural bases of interpersonal influence in groups: A longitudinal case study. American Sociological Review, 58, 861-872.

Gilsing, V., Nooteboom, B., van Haverbeke, W., Duysters, G., \& van den Oord, A. (2008). Network embeddedness and the exploration of noveltechnologies: Technological distance, betweenness centrality and density. Research Policy, 28, 1717-1731.

Girmscheid, G. (2007). Projektabwicklung in der Bauwirtschaft. Berlin: Springer-Verlag.

Girtler, R. (2001). Methoden der qualitativen Sozialforschung. Köln: Böhlan.

Heuskel, D. (1999). Wettbewerb jenseits von Industriegrenzen: Aufbruch zu neuen Wachstumsstrategien. Frankfurt a. M.: Campus Verlag.

Johnston, R., \& Lawrence, P. R. (1998). Beyond vertical integration - the rise oft he value adding partnership. In: Markets, Hierarchies \& Networks: the coordination of social life, (Hrsg.), Thompson et al. Sage Publication, London.

Jones, G. R., \& George, J. M. (1998). The experience and evolution of trust: Implications for cooperation and teamwork. Academy of Management, 23, 531-546.

Knop, R. (2009). Erfolgsfaktoren strategischer Netzwerke kleiner und mittlerer Unternehmen. Wiesbaden: Gabler.

Krippendorff, K. (1980). Content Analysis: An introduction to its methodology. London: Sage.

Larson, A. (1992). Network dyads in entrepreneurial settings: A study of the governance of exchange relationships. Administrative Science Quarterly, 37, 76-104.

Love, P. E. D., Gunasekaran, A., \& Li, H. (1998). Concurrent engineering: A strategy for procuring construction projects. International Journal of Project Management, 16, 375-383.

Mayring, P. (2000). Qualitative Inhaltsanalyse: Grundlagen und Techniken. (7. Aufl.). Weinheim: Beltz Verlag.

Mayring, P. (2002). Einführung in die qualitative Sozialforschung: Eine Anleitung zu qualitativem Denken (5. Aufl.). Weinheim: Beltz Verlag.

Miller, D. (1981). Towards a new contingency approach: The search of organizational gestalts. Journal of Management Studies, 18, 1-21.

Miller, D., \& Mintzberg, H. (1983). The case for configuration. In G. Morgen (Hrsg.), Beyond method (S. 57-73). Beverly Hills: Sage.

Meixner, O., \& Haas, R. (2002). Computergestützte Entscheidungsfindung. Frankfurt a. M.: Redline Wirtschaft bei Ueberreuter.

Möller, K. (2006). Wertschöpfung in Netzwerken. München: Vahlen. 
Olsen, B. E., Haugland, S. A., Karlsen, E., \& Husoy, G. J. (2005). Governance of complex procurements in the oil and gas industry. Journal of Purchasing \& Supply Management, 11, 5-20.

Pfnür, A. (2002). Betriebliche Immobilienökonomie. Heidelberg: Physica-Verlag.

Pfnür, A. (2011). Modernes Immobilienmanagement (3. Aufl.). Berlin: Springer.

Pinto, J., \& Mantel, S. (1990). The causes of project failure. IEEE Transactions on Engineering Management, 37(4), 269-276.

Prahalad, C. K., \& Hamel, G. (1990). The core competence of the corporation. Harvard Business Review, May-June 1990, S. 79-91.

Provan, K. G., Fish, A., \& Sydow, J. (2007). Interorganizational networks at the network level: A review of the empirical literature on whole networks. Journal of management, 33, 479-516.

Saaty, T. L. (1986). Axiomatic foundation of the analytic hierarchy process. Management Science, 32, $841-855$.

Shepherd, C., \& Ahmed, P. K. (2000). From product innovation to solutions innovation: A new paradigm for competitive advantage. European Journal of Innovation Management, 3(2), 100-106.

Sydow, J. (1992). Strategische Netzwerke: Evolution und Organisation. Wiesbaden: Gabler.

Sydow, J., \& Van Well, B. (2010). Wissensintensiv durch Netzwerkorganisation. In: J. Sydow (Hrsg.), Management von Netzwerkorganisationen. Beiträge aus der Managementforschung (5. Aufl.). Wiesbaden: Gabler Verlag.

Teece, D. J. (1986). Profiting from technological innovation: Implications for integration, collaboration, licensing and public policy. Research Policy, 15, 785-805.

Tsai, W. (2002). Social structure of "Coopetition" within a multiunit organization: Coordination, competition, and intraorganizational knowledge sharing. Organization Science, 13, 179-190.

Uhl, S., \& Höppner, G. (2010). Effizienzsteigerungen im öffentlichen Bau durch funktionale Ausschreibungen. Erlangen: Friedrich Alexander Universität.

Uzzi, B. (1997). Social structure and competition in interfirm networks: The paradox of embeddedness. Administrative Science Quarterly, 42, 35-67.

Wasko, M. M., \& Faraj, S. (2005). Why should I share? Examining social capital and knowledge contribution, MIS Quarterly, 29, 35-57.

Wasserman, S., \& Faust, K. (1994). Social network analysis: Methods and applications. Cambridge: Cambridge University Press. 